\title{
Association between bone marrow fluorodeoxyglucose uptake and recurrence after curative surgical resection in patients with T1-2NOMO lung adenocarcinoma: a retrospective cohort study
}

\author{
Tian-Cheng $\mathrm{Li}^{1}$, Li-Li Wang ${ }^{2}$, Bo-Le Liu ${ }^{2}$, Jun-Jie Hong ${ }^{2}, \mathrm{Ni}-\mathrm{Na} \mathrm{Xu}^{2}$, Kun Tang ${ }^{2 \#}$, Xiang-Wu Zheng ${ }^{2 \#}$ \\ ${ }^{1}$ PET Center, The First Affiliated Hospital, College of Medicine, Zhejiang University, Hangzhou, China; ${ }^{2}$ Department of PET/CT, Radiology \\ Imaging Center, The First Affiliated Hospital of Wenzhou Medical University, Wenzhou, China
}

\#These authors contributed equally to this work.

Correspondence to: Xiang-Wu Zheng, MD; Kun Tang, PhD. The First Affiliated Hospital of Wenzhou Medical University, Xuefu North Road, Wenzhou 325000, China. Email: zxwu111@sina.com; kuntang007@163.com.

Background: Evidence regarding the relationship between fluorodeoxyglucose (FDG) uptake in the bone marrow of patients with lung adenocarcinoma and prognosis is limited. This study aimed to identify whether bone marrow FDG uptake is a risk factor for recurrence in patients after curative surgical resection of T12N0M0 lung adenocarcinoma.

Methods: From January 2012 to December 2016, we retrospectively enrolled 195 pT1-2N0M0 lung adenocarcinoma patients who underwent both preoperative FDG positron emission tomography/computed tomography (PET/CT) and surgical resection from the lung adenocarcinoma database maintained by the PET/CT department at our hospital. After surgical resection, patients were followed up mainly through regular outpatient examinations. The maximum standardized uptake value $\left(\mathrm{SUV}_{\max }\right)$ of the primary tumor, the mean FDG uptake of bone marrow (BM SUV), bone marrow-liver uptake ratio (BLR), metabolic tumor volume (MTV), and total lesion glycolysis (TLG) were measured from the pretreatment FDG PET/CT images. Multi-adjusted Cox proportional hazards models were built to evaluate the independent prognostic value of BLR in predicting recurrence-free survival (RFS). A restricted cubic spline regression model was conducted to provide more precise estimates and examine the shape of the associations between BLR and the risk of recurrence.

Results: The follow-up results showed that 30 of the 195 patients (15.4\%) had tumor recurrence. Compared with non-recurrent patients, the primary tumor size in recurrent patients was larger, and the $\mathrm{SUV}_{\max }$, TLG, and serum C-reactive protein (CRP) levels were higher. Univariate analysis showed that BLR, tumor size, $\mathrm{SUV}_{\max }$, TLG, and CRP were significantly correlated with postoperative tumor recurrence. After adjustment for conventional confounding factors, the hazard ratio of BLR was 5.01 (95\% CI, 1.32, 18.98) for the highest tertile of BLR compared with the lowest tertile. The multi-adjusted spline regression showed that BLR had a linear relationship with $\log$ relative risk (RR) for recurrence when BLR was lower than 0.7. Over this level, the effect stabilized, suggesting a saturation effect for BLR at a level of approximately 0.7 at recurrence.

Conclusions: BLR was an independent risk factor for predicting RFS in T1-2N0M0 lung adenocarcinoma patients after curative surgical resection. BLR can be used as a biomarker for evaluating the risk of lung cancer recurrence.

Keywords: Lung adenocarcinoma; recurrence; positron emission tomography (PET); fluorodeoxyglucose (FDG); bone marrow $(\mathrm{BM})$ 
Submitted Nov 18, 2019. Accepted for publication Aug 06, 2020.

doi: 10.21037/qims-19-962

View this article at: http://dx.doi.org/10.21037/qims-19-962

\section{Introduction}

Lung cancer remains the leading cause of cancer-related death worldwide. Non-small cell lung cancer (NSCLC) accounts for approximately $83 \%$ of all lung cancer $(1,2)$. At present, curative surgical resection offers the best prognosis for NSCLC patients. However, even with curative surgical resection and no malignant lymph node metastasis, approximately $20 \%$ of T1-2N0M0 lung cancer patients still have recurrence at follow-up (3). If patients with T1-T2N0M0 lung adenocarcinoma have a high risk of recurrence, they may benefit from more aggressive tailored treatments, such as postoperative chemotherapy. Therefore, differentiating patients who have a high risk of postoperative recurrence is critical for the selection of further adjuvant therapy. Due to the poor prognosis of NSCLC, research into various clinical factors for the prediction of NSCLC and the selection of appropriate auxiliary indexes are critical. Neutrophil-lymphocytes ratio (NLR), plateletlymphocyte ratio (PLR), albumin and C-reactive protein (CRP), and other systemic inflammatory markers have been demonstrated to be important prognostic factors for predicting clinical outcomes (4-8).

In addition to serum inflammatory markers, several recent studies have identified imaging biomarkers of systemic inflammatory responses using F-18 fluorodeoxyglucose (FDG) positron emission tomography/computed tomography (PET/CT) (9-11). PET/CT uses FDG as a molecular imaging agent, which has been widely used in the diagnosis, staging, evaluation of treatment efficacy, and prognosis of NSCLC (12-14). Several studies have shown that the uptake of FDG in the bone marrow (BM) of patients with lung cancer is significantly higher than that in patients with benign pulmonary nodules (15). Furthermore, FDG uptake in the $\mathrm{BM}$ of patients with malignant tumors has been significantly associated with serum inflammatory markers, suggesting that FDG uptake of the BM can reflect systemic inflammatory responses $(16,17)$. However, the prognostic value of $\mathrm{BM}$ FDG uptake in T1-2N0M0 lung adenocarcinoma patients remains unclear. The purpose of this study was to evaluate the association between FDG uptake in BM on PET/CT and recurrence after curative surgical resection in patients with T1-2N0M0 lung adenocarcinoma.

\section{Methods}

\section{Patients}

The Institutional Ethics Committee of the First Affiliated Hospital of Wenzhou Medical University approved this retrospective study, and the requirement to obtain informed consent was waived. From January 2012 to December 2016, 195 consecutive pT1-2N0M0 lung adenocarcinoma patients identified from the lung adenocarcinoma database maintained by the PET/CT department were included in this study. All patients received blood tests and FDG PET/CT imaging before surgery. Patients were excluded from the study according to the following criteria: (I) patients had received radiotherapy, chemotherapy and targeted therapy before or after surgery; (II) patients had a history of another malignancy or chronic liver disease; (III) patients showed moderate-to-severe anemia (hemoglobin $<10.0 \mathrm{~g} / \mathrm{dL}$ ) in preoperative blood tests or had coexisting acute inflammatory disease; (IV) patients had an injection of erythropoietin or granulocyte-macrophage colonystimulating factor (GM-CSF) within 6 months before FDG PET/CT; (V) patients had pathological bone marrow infiltration confirmed by bone marrow biopsy or by a whole-body MRI; (VI) patients were lost to clinical followup within 12 months after surgical resection.

All patients underwent lobectomy, bilobectomy, or pneumonectomy with systematic lymph node dissection according to their stage and clinical condition after the preoperative examination. After curative surgery, all patients underwent regular clinical follow-up including physical examination, blood tests and CT or magnetic resonance imaging (MRI) scans every 3-6 months. In cases with abnormal findings on follow-up examinations, additional imaging studies including contrast-enhanced CT or MRI, PET/CT scans, and histological confirmation were performed to verify tumor recurrence. The followup period ranged from 3.5 to 65 months. Recurrencefree survival (RFS) was defined as the time from surgery to the first diagnosis of local, regional or distant disease recurrence, or the last follow-up. Patients who were lost to follow-up were considered a censoring event for recurrence. 


\section{FDG PET/CT protocol}

Patients fasted at least 6 hours to ensure that the blood glucose concentration was not higher than $110 \mathrm{mg} / \mathrm{dL}$ just before the injection of FDG. FDG PET/CT images were acquired using a body PET/CT scanner (GEMINI TF 64; Philips, The Netherlands) and scanning was performed approximately 60 minutes after intravenous (IV) injection of FDG with a dosage of $3.7 \mathrm{MBq} / \mathrm{kg}$. A low-dose unenhanced CT scan was performed from the skull base to the middle of the thigh with the following parameters: $120 \mathrm{kV}, 80 \mathrm{~mA}$, a pitch of 0.829 , a tube rotation time of 0.5 second per rotation, and a reconstruction thickness and interval of $5.0 \mathrm{~mm}$ for precise anatomical localization and attenuation correction. This was followed by a PET scan that matched the CT section thickness. PET images were obtained using the ordered subset expectation maximization method. Images were transferred into Philips EBW 3.0 to reconstruct PET, CT, and PET/CT fusion images.

\section{Imaging analysis}

PET/CT images were interpreted by an experienced radiologist using the fixed threshold method on the MEDEX workstation system, and the regions of interest (volume of interest, VOI) were manually drawn on the original site of the tumor. For lesions with respirationinduced artifacts, we manually moved the PET or CT images up, down, left, and right to make them match each other as much as possible in the PET/CT fusion images. The maximum standard uptake value within the VOI $\left(\mathrm{SUV}_{\max }\right)$ and the average SUV of voxels within the VOI exceeding $40 \%$ of the $\mathrm{SUV}_{\text {max }}\left(\mathrm{SUV}_{\text {avg }}\right.$ ) were automatically measured. The tumor volume of a fixed threshold of $40 \% \mathrm{SUV}_{\max }$ or greater was measured and defined as the metabolic tumor volume (MTV). Total lesion glycolysis (TLG) was calculated by multiplying the MTV and $\mathrm{SUV}_{\text {avg }}$ of the tumor. Subsequently, PET/CT parameters of the BM including BM SUV and bone marrow-liver uptake ratio (BLR) were measured. Spheroid-shaped VOIs were manually drawn over the vertebral body of each of the six vertebrae in the thoracic and lumbar spine. Spines that showed compression fractures, severe osteoarthritic change, or post-operative change by spinal surgery were excluded from measurement. Within each VOI in the vertebral body, an automatic isocontour set at $75 \%$ of the maximum SUV was automatically generated, and the mean SUV of voxels within the isocontour was calculated. This method, using a cutoff value of $75 \%$ of the maximum SUV, has demonstrated good reproducibility for measuring FDG uptake of the BM in a previous study (18). The mean SUV of the VOIs in the six selected vertebrae was calculated and defined as the BM SUV. Two $2 \mathrm{~cm}$-sized spheroidal VOIs were drawn both in the right lobe and the left lobe of the liver, and the mean SUV of the normal liver tissue was also measured. To calculate the BLR for each patient, the BM SUV was divided by the mean SUV of the normal liver tissue.

\section{Statistical analysis}

NLR and PLR were calculated for each patient after blood testing. To assess the differences in baseline characteristics according to the BLR stratification, continuous variables were evaluated using the Kruskal-Wallis test and categorical variables were evaluated using Pearson's Chi-square test or Fisher's exact test as appropriate. The Mann-Whitney $\mathrm{U}$ test was used for the comparison of variables between patients with or without recurrence. To assess the predictive values of variables for RFS, univariate and multivariate analyses were performed using a Cox proportional hazards regression model. To ensure the robustness of data analysis, we performed a series of sensitivity analyses where we converted the BLR into a categorical variable and calculated the $\mathrm{P}$ value for trends. The purpose was to verify the results of BLR as a continuous variable and to observe the possibility of nonlinearity. Hazard ratios with Wald 95\% CI were provided for the univariate and multivariate analyses. For BLR, survival curves were estimated using the KaplanMeier method, and compared using the log-rank test. A restricted cubic spline regression model was conducted to provide more precise estimates and examine the shape of the associations between BLR as a continuous variable and the risk of recurrence. $\mathrm{P}<0.05$ was considered statistically significant. All analyses were performed with the statistical software packages, R (http://www.R-project.org, The R Foundation) and EmpowerStats (http://www.empowerstats. com, X\&Y Solutions, Inc., Boston, MA, USA).

\section{Results}

\section{Patient characteristics}

The characteristics of the enrolled patients are shown in Table 1. A total of 195 patients with T1-2N0M0 lung adenocarcinoma were included in this study, including 
Table 1 Characteristics of all patients

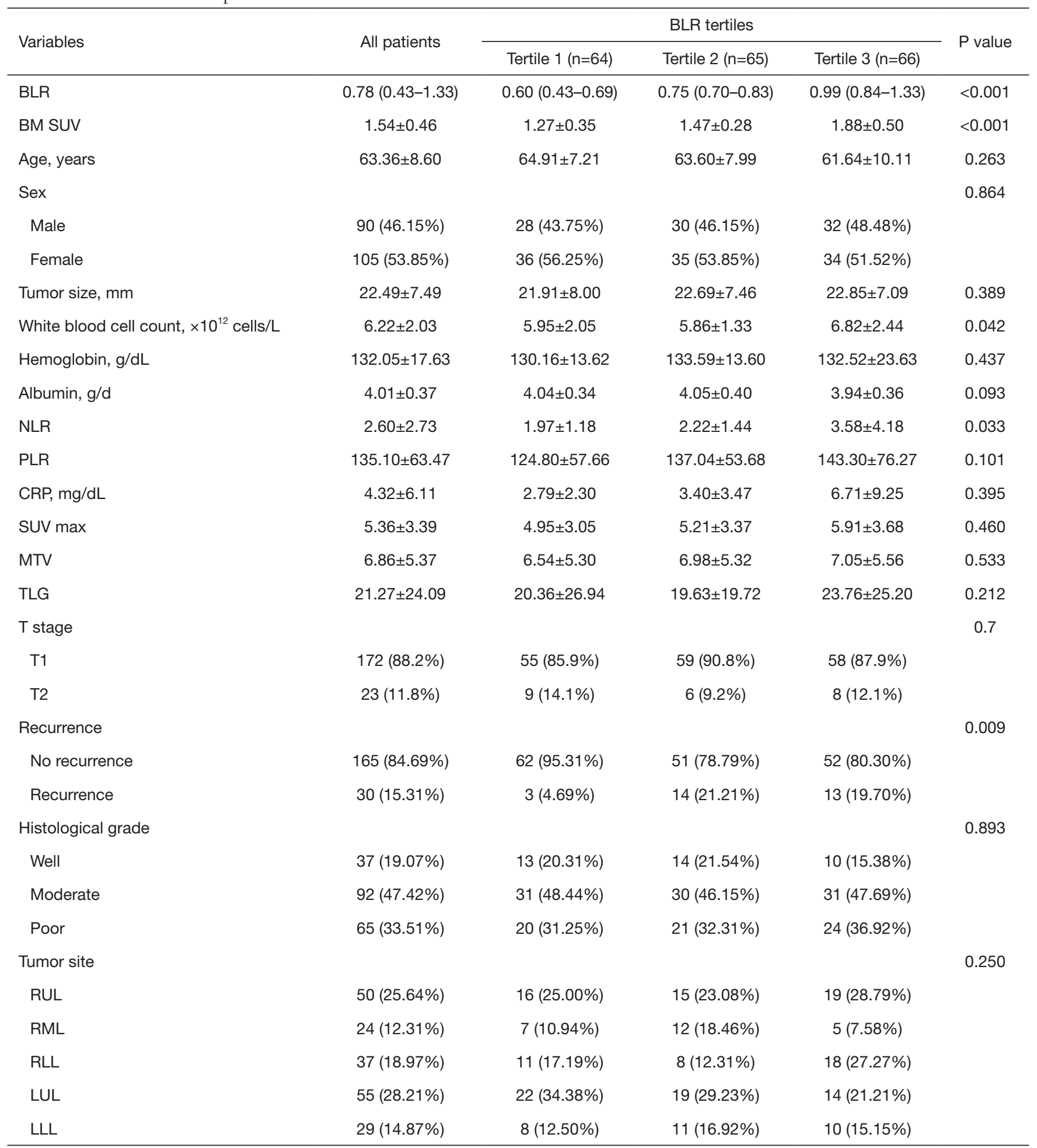

BLR, bone marrow to liver uptake ratio; BM SUV, mean standardized uptake value of bone marrow; CRP, C-reactive protein; NLR, neutrophil-lymphocyte ratio; PLR, platelet lymphocyte ratio; SUVmax: maximum standardized uptake value of primary tumor; MTV, metabolic tumor volume; TLG, total lesion glycolysis; RUL, right upper lobe; RML, right middle lobe; RLL, right lower lobe; LUL, left upper lobe; LLL, left lower lobe. 
90 males and 105 females, with an average age of $63.36 \pm 8.60$ years. Of the 195 enrolled patients, 30 (15.4\%) patients experienced disease progression during the clinical follow-up. The mean RFS was 21.5 months. Patients were divided into three groups according to the tertiles of the BLR level. The cut-off values and groups according to the tertiles of BLR were T1 (0.43-0.69), T2 (0.70-0.83), and T3 (0.84-1.33). Patients with low, middle, and high BLR values were similar in most characteristics except for white blood cell (WBC) count and NLR. Higher BLR tertiles were significantly associated with higher recurrence risk than the lowest tertiles $(\mathrm{P}=0.009)$. Compared with nonrecurrent patients, recurrent patients had larger tumor size and higher CRP, SUV $V_{\max }$, and TLG values. Detailed patient characteristics are described in Table 2. Figure 1 shows a patient who had increased FDG uptake of bone marrow before surgery and experienced a recurrence during follow-up.

\section{Association between the level of BLR and recurrence}

The age, sex, histological grade, tumor size, serum albumin, hemoglobin, WBC, NLR, PLR, CRP, SUV $\max _{\max }$ MTV, TLG, BM SUV, and BLR values of all patients were evaluated in the univariate analysis. CRP, BLR, tumor size, tumor $\mathrm{SUV}_{\max }$, and TLG were significantly associated with postoperative recurrence (Table 3).

The multivariate model clarified the relationship between BLR and recurrence regardless of whether the variables were adjusted or not (Table 4). Model 1 was adjusted by sex and age, and model 2 was further adjusted by tumor size, hematological parameters (CRP), and PET parameters $\left(\mathrm{SUV}_{\text {max }}\right.$, TLG) based on model 1. For the sensitivity analysis, we converted the BLR from a continuous variable to a categorical variable (tertile of BLR). The $\mathrm{P}$ trend of BLR as a categorical variable in the fully adjusted model was inconsistent with the result when BLR was a continuous variable. Moreover, we used restricted cubic spline regression to confirm the nonlinear relationships between continuous BLR levels and recurrence (Figure 2). The spline regression showed that BLR had a linear relationship with log relative risk (RR) for recurrence when BLR was lower than 0.7. Over this value, the effect became stable.

\section{Kaplan-Meier survival analysis}

For Kaplan-Meier survival analysis (Figure 3), BLR was divided into three categories according to the tertiles of the BLR level. Kaplan-Meier analysis of patients stratified by BLR value showed significantly different RFS rates between the three groups of patients $(\mathrm{P}=0.0029)$. The patients in the middle and high BLR groups had a significantly worse RFS than those in the low BLR group.

\section{Discussion}

In the present study, BLR, which was defined as the ratio of bone marrow FDG uptake to liver, was used to reduce the interindividual variation of BM SUV (17). The present study demonstrated that BLR is an independent risk factor for recurrence in patients with T1-2N0M0 lung adenocarcinoma. In the multivariate recurrence model, which included tumor size, PET/CT parameters of the primary tumor $\left(\mathrm{SUV}_{\text {max }}\right)$, and serum inflammatory markers (CRP), BLR was found to be the independent prognostic factor for RFS when treated as a categorical variable. The multi-adjusted spline regression showed that BLR had a linear relationship with $\log \mathrm{RR}$ for recurrence when BLR was lower than 0.7. Above this level, the effect remained stable, which suggested a saturation effect for BLR at a level of approximately 0.7 at recurrence.

There are many risk factors for cancer prognosis. The prognosis of patients depends not only on the primary tumor and the clinical characteristics of patients but also on the tumor microenvironment. Previous studies have shown that inflammatory response markers play an important role in the occurrence and development of cancer $(19,20)$. Neutrophils, lymphocytes, and platelets in peripheral blood cause stress reactions during the process of inflammation, which may cause oxidative damage to cells and change the state of the tumor microenvironment. These factors can promote the transformation, growth, invasion, and metastasis of tumor cells (21). NLR is an important prognostic index in patients with NSCLC as an indicator of the inflammatory response, and can be used as a potential biomarker in immunotherapy and radiotherapy (22). Platelets play an important role in hemostasis, thrombosis, and inflammation. In recent years, the interaction between platelets and tumor parameters such as tumor growth, 
Table 2 Clinical and demographic characteristics of patients with recurrence and non-recurrence

\begin{tabular}{|c|c|c|c|}
\hline Recurrence & No recurrence & Recurrence & $P$ value \\
\hline Age, years & $63.59 \pm 8.53$ & $62.13 \pm 9.05$ & 0.459 \\
\hline Tumor size, mm & $22.00 \pm 7.21$ & $25.17 \pm 8.51$ & $0.033^{*}$ \\
\hline White blood cell count, $\times 10^{12}$ cells $/ L$ & $6.23 \pm 2.03$ & $6.14 \pm 2.10$ & 0.787 \\
\hline PLR & $135.81 \pm 66.32$ & $131.23 \pm 45.50$ & 0.875 \\
\hline CRP, mg/dL & $3.96 \pm 6.06$ & $6.32 \pm 6.13$ & $0.013^{*}$ \\
\hline Hemoglobin, g/dL & $131.49 \pm 18.38$ & $135.13 \pm 12.56$ & 0.165 \\
\hline Albumin, g/d & $4.02 \pm 0.37$ & $3.95 \pm 0.39$ & 0.317 \\
\hline TLG & $19.55 \pm 22.96$ & $30.68 \pm 28.14$ & $0.001^{*}$ \\
\hline BM SUV & $1.53 \pm 0.47$ & $1.60 \pm 0.43$ & 0.449 \\
\hline BLR & $0.77 \pm 0.18$ & $0.82 \pm 0.16$ & 0.085 \\
\hline Sex & & & 0.462 \\
\hline Male & $78(47.27 \%)$ & $12(40.00 \%)$ & \\
\hline Female & $87(52.73 \%)$ & $18(60.00 \%)$ & \\
\hline Histological grade & & & 0.387 \\
\hline Well & 34 (20.61\%) & $3(10.00 \%)$ & \\
\hline RLL & 32 (19.39\%) & 5 (16.67\%) & \\
\hline LUL & 46 (27.88\%) & 9 (30.00\%) & \\
\hline LLL & 26 (15.76\%) & $3(10.00 \%)$ & \\
\hline
\end{tabular}

*, significance. BLR, bone marrow to liver uptake ratio; BM SUV, mean standardized uptake value of bone marrow; CRP, C-reactive protein; NLR, neutrophil-lymphocyte ratio; PLR, platelet lymphocyte ratio; SUVmax, maximum standardized uptake value of primary tumor; MTV, metabolic tumor volume; TLG, total lesion glycolysis; RUL, right upper lobe; RML, right middle lobe; RLL, right lower lobe; LUL, left upper lobe; LLL, left lower lobe.

angiogenesis, and tumor metastasis has been the subject of much investigation. PLR can reflect a certain degree of inflammation in the body and has a negative relationship with prognosis (23). However, in this study, PLR and NLR showed no prognostic value in the univariate analysis. This may due to the differences in the enrolled populations or institutional-based bias. It has also been reported that peripheral blood NLR is not an independent prognostic factor for patient survival, and that the level of NLR in peripheral blood is influenced by other factors leading to poor reliability (24).

Because of the significant correlation between FDG 

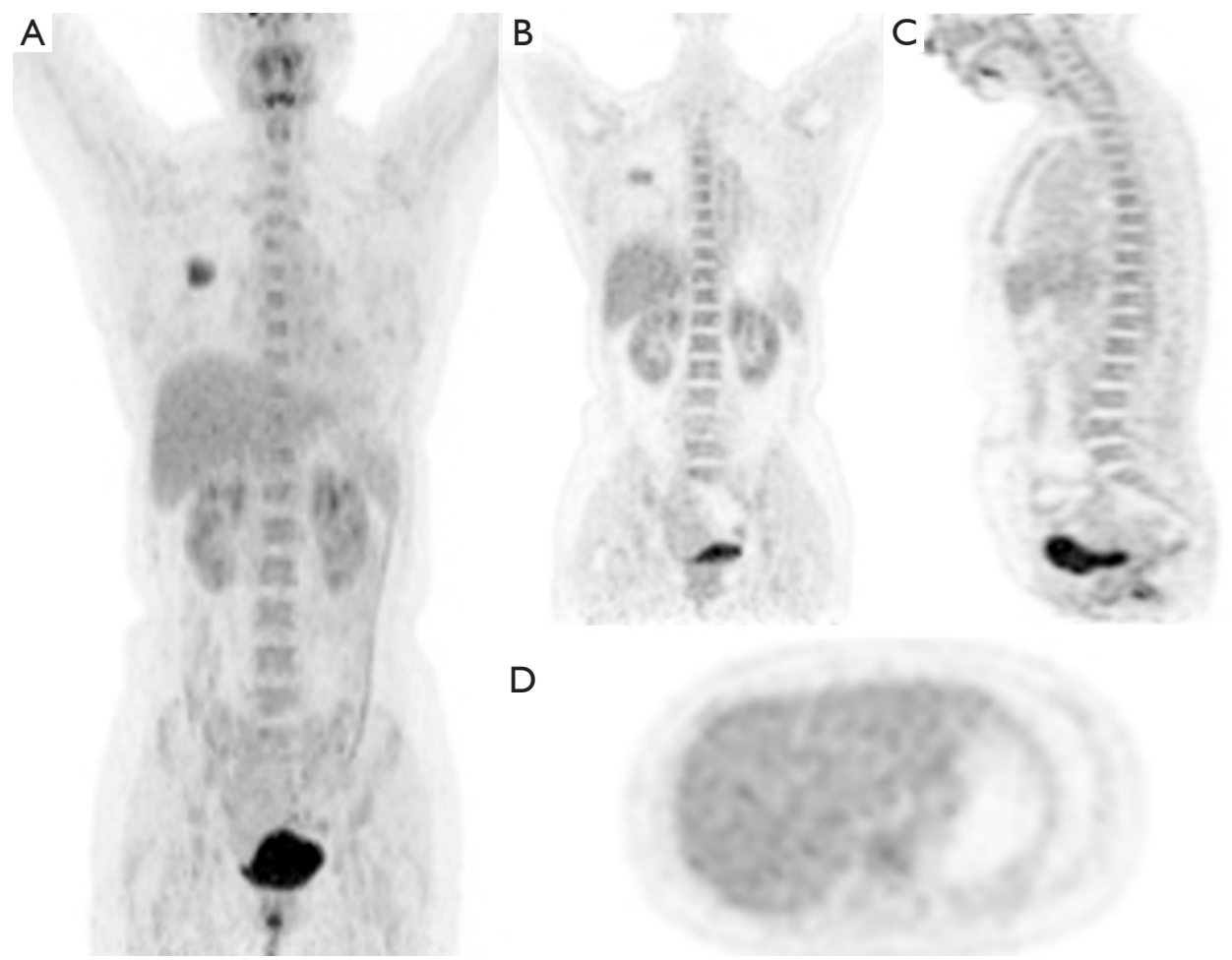

Figure 1 Representative PET images of one patient with relatively high BLR level. (A) Maximum intensity projection, (B) coronal, (C) sagittal, and (D) transaxial fluorodeoxyglucose (FDG) positron emission tomography (PET) images of a 51-year-old woman with lung adenocarcinoma. Increased FDG uptake in the BM is shown, and the BM to liver uptake ratio was 1.13. Cancer recurred 15 months after curative surgical resection. BM, bone marrow; FDG, fluorodeoxyglucose; PET, positron emission tomography.

uptake in BM and serum inflammatory markers, several studies have focused on the evaluation of the prognostic value of BM SUV and BLR for lung cancer patients. In a meta-analysis of 15 studies across 1,197 patients, Jeong et al. found that, compared to patients with a high level of bone marrow uptake, patients with low level FDG bone marrow uptake had better event-free survival rates and overall survival rates. Similar findings were also reported in a study by Lee $e t$ al., where the relationship between bone marrow SUV and prognosis in patients with NSCLC after curative surgical resection was analyzed (18). In their study, BLR was a significant prognostic factor for recurrence in the multivariate analysis, consistent with the results of our study. However, the patients included in their study had I-III stage lung cancer, and received various kinds of treatments. In contrast, only T1-2N0M0 lung adenocarcinoma patients without postoperative chemotherapy or radiotherapy who underwent curative surgical resection were recruited in our study. Lee $e t a l$. also investigated the prognostic significance of BM SUV in patients with other kinds of cancer, including head and neck squamous cell carcinoma (HNSCC), gastric cancer, and colorectal cancer $(10,25,26)$. Their research also found that BM SUV or BLR was an independent predictor of prognosis in these cancers. However, their studies did not explore if there was a nonlinear relationship between BM SUV and prognosis, and did not measure the targetindependent variable as both a continuous variable and categorical variable, which can enhance the robustness of results.

In our study, $15.3 \%$ of lung adenocarcinoma patients presented with recurrence during follow-up, which is similar to the recurrence incidence reported in a previous study (27). Compared with non-recurrent patients, the size of the primary tumor of patients with recurrence was larger, and the CRP, SUV $\mathrm{max}_{\max }$, and TLG values were higher. Kaplan-Meier analysis showed that patients with relatively high BLR values had a significantly higher recurrence rate than patients with low BLR values, which was also consistent with previous studies. The main advantage of this study was that we used restricted cubic spline regression to 
Table 3 Univariate analysis for recurrence

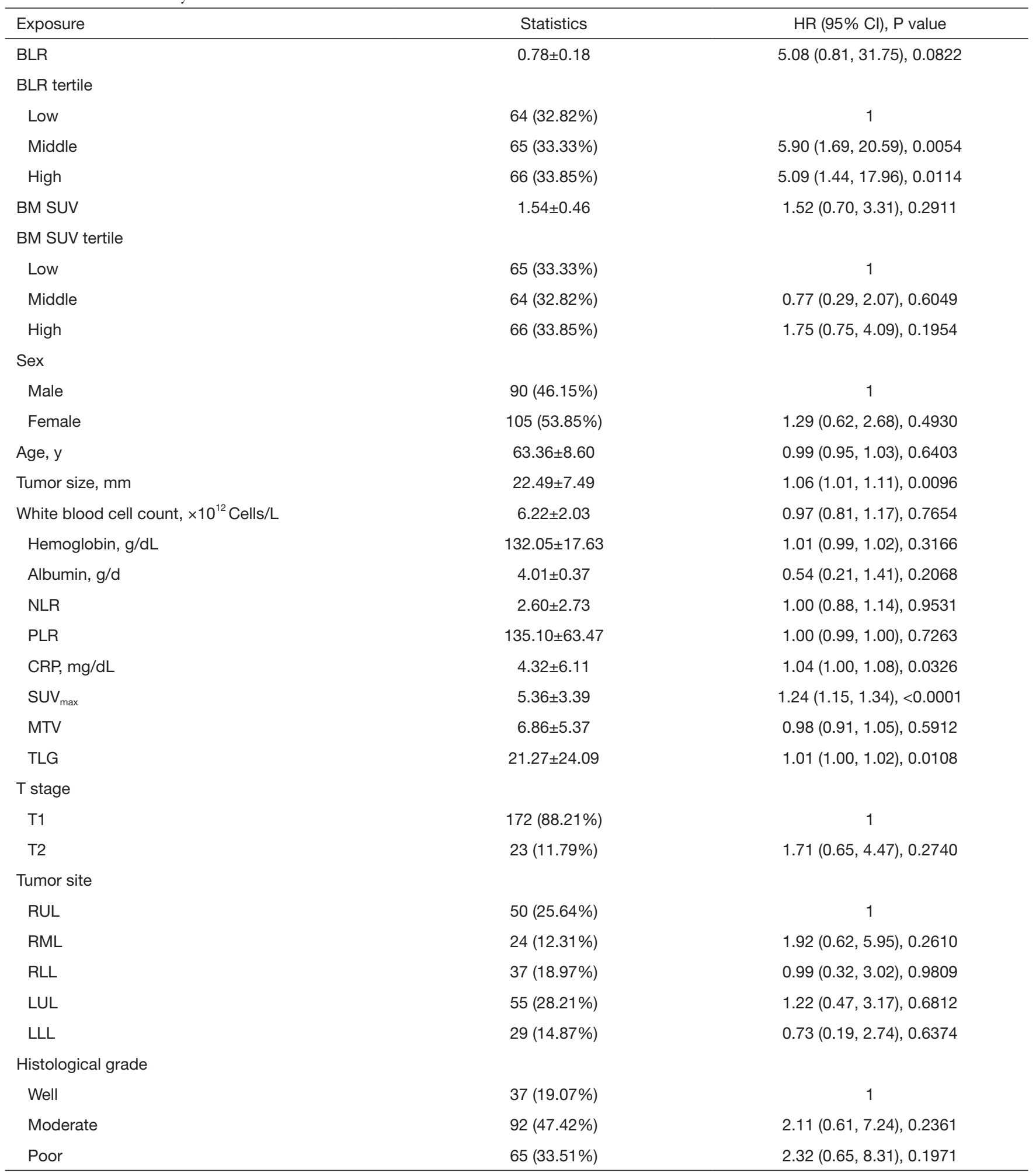

$\mathrm{HR}$, hazard ratio; $\mathrm{Cl}$, confidence interval; BLR, bone marrow to liver uptake ratio; BM SUV, mean standardized uptake value of bone marrow; CRP, C-reactive protein; NLR, neutrophil-lymphocyte ratio; PLR, platelet lymphocyte ratio; SUVmax, maximum standardized uptake value of primary tumor; MTV, metabolic tumor volume; TLG, total lesion glycolysis; RUL, right upper lobe; RML, right middle lobe; RLL, right lower lobe; LUL, left upper lobe; LLL, left lower lobe. 
Table 4 Relationship between BLR and recurrence using different models from multivariate analysis

\begin{tabular}{lccc}
\hline & Crude model HR $(95 \% \mathrm{Cl}), \mathrm{P}$ value & Model I HR (95\% Cl), P value & Model II HR (95\% Cl), P value \\
\hline BLR & $5.08(0.81,31.75), 0.0822$ & $5.09(0.80,32.23), 0.0839$ & $5.85(0.72,47.40), 0.0981$ \\
BLR Tertile & 1.0 & 1.0 & 1.0 \\
Low & $5.90(1.69,20.59), 0.0054$ & $6.03(1.72,21.12), 0.0050$ & $7.16(1.96,26.23), 0.0029$ \\
Middle & $5.09(1.44,17.96), 0.0114$ & $5.22(1.47,18.57), 0.0108$ & $5.01(1.32,18.98), 0.0177$ \\
High & 0.0141 & 0.0133 & 0.0337 \\
\hline
\end{tabular}

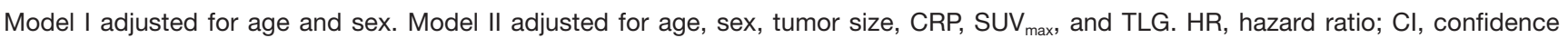
interval; BLR, bone marrow to liver uptake ratio; CRP, C-reactive protein; SUV $_{\max }$, maximum standardized uptake value of primary tumor; TLG, total lesion glycolysis.

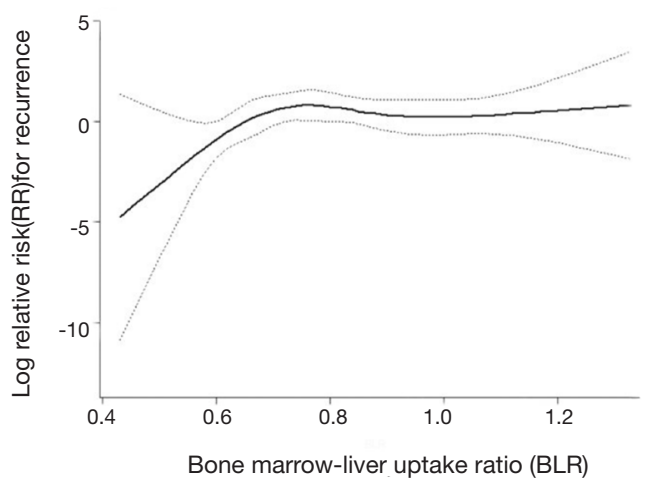

Figure 2 Association between bone marrow-liver uptake ratio (BLR) levels with risk of recurrence. Dashed lines are 95\% confidence intervals. Relative risk (RR) and $95 \%$ confidence intervals were derived from restricted cubic spline regression. Relative risk was estimated using cox regression modeling, adjusting for the same variables as model 2 in Table 4.

highlight a saturation effect between BLR and recurrence risk after adjusting for various confounding factors. The results showed that when BLR was higher than 0.7, the risk of recurrence no longer increased. Overall, a better understanding of the role of BLR can help clinicians predict the recurrence of early-stage lung cancer and select more appropriate treatment for patients.

This study had several limitations. First, the study was a retrospective single-center study with a relatively small number of patients. Further studies using a larger number of patients are needed to validate the results of this study. Second, we only enrolled patients with predominantly stage I lung adenocarcinoma according to the $8^{\text {th }}$ edition of the lung cancer clinical staging system (28); hence, our results cannot be extrapolated to other populations. Additionally, our study only included traditional PET parameters in analysis, and other researchers have attempted to mine the radiomics features of PET (29,30); we will focus on this in future studies. Finally, considering that the role of inflammation in cancer is still poorly understood, further studies are needed to explore and evaluate more inflammatory markers.

In conclusion, BLR was an independent risk factor for predicting RFS in T1-2N0M0 lung adenocarcinoma patients after curative surgical resection. Patients with low BLR values had a better prognosis than those with high BLR values. Furthermore, in multi-adjusted restricted cubic spline regression, BLR as a continuous variable showed a nonlinear relationship with recurrence risk. Therefore, BLR can be used as a biomarker for stratifying the risk of lung cancer recurrence. 


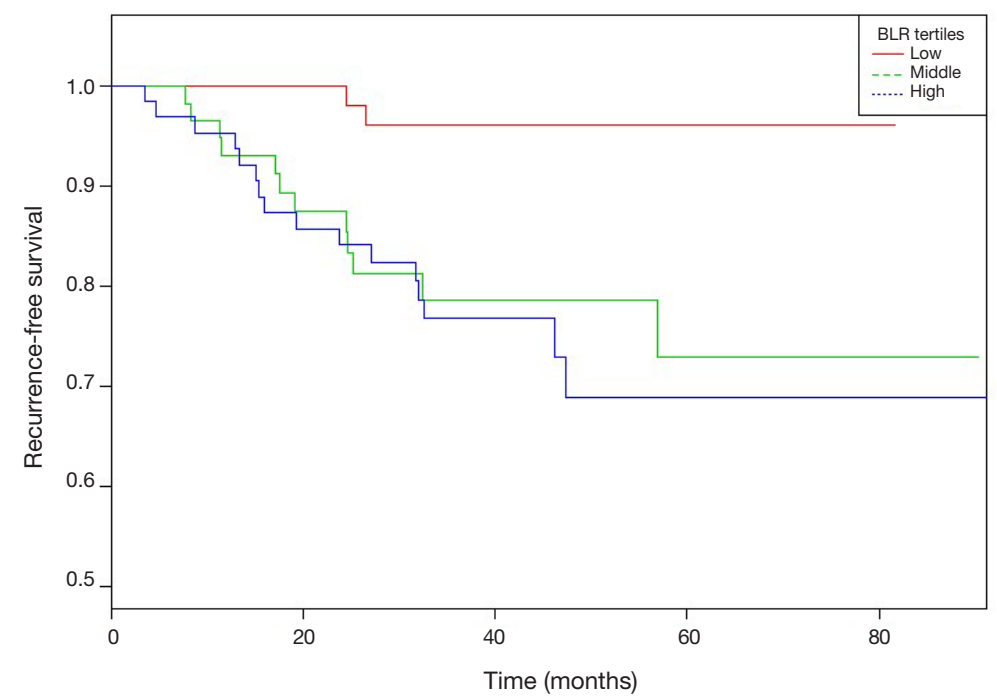

Figure 3 Cumulative recurrence-free survival (RFS) curves according to bone marrow-liver uptake ratio (BLR) in the 195 enrolled patients. BLR was divided into three categories according to the tertiles of the BLR level. Kaplan-Meier analysis of patients stratified by BLR value showed significantly different RFS rates between the three groups of patients $(\mathrm{P}=0.0029)$. The patients in the middle and high BLR groups had a significantly worse RFS than those in the low BLR group.

\section{Acknowledgments}

Funding: This work was supported by Zhejiang province public welfare technology application research project (No. LGF19H180012).

\section{Footnote}

Conflicts of Interest: All authors have completed the ICMJE uniform disclosure form (available at http://dx.doi. org/10.21037/qims-19-962). The authors have no conflicts of interest to declare.

Ethical Statement: This retrospective study was approved by the Institutional Ethics Committee of the First Affiliated Hospital of Wenzhou Medical University, and the requirement to obtain informed consent was waived.

Open Access Statement: This is an Open Access article distributed in accordance with the Creative Commons Attribution-NonCommercial-NoDerivs 4.0 International License (CC BY-NC-ND 4.0), which permits the noncommercial replication and distribution of the article with the strict proviso that no changes or edits are made and the original work is properly cited (including links to both the formal publication through the relevant DOI and the license).
See: https://creativecommons.org/licenses/by-nc-nd/4.0/.

\section{References}

1. Siegel RL, Miller KD, Jemal A. Cancer statistics, 2019. CA Cancer J Clin 2019;69:7-34.

2. Miller KD, Nogueira L, Mariotto AB, Rowland JH, Yabroff KR, Alfano CM, Jemal A, Kramer JL, Siegel RL. Cancer treatment and survivorship statistics, 2019. CA Cancer J Clin 2019;69:363-85.

3. Kiankhooy A, Taylor MD, LaPar DJ, Isbell JM, Lau CL, Kozower BD, Jones DR. Predictors of early recurrence for node-negative t 1 to $\mathrm{t} 2 \mathrm{~b}$ non-small cell lung cancer. Ann Thorac Surg 2014;98:1175-83.

4. Diem S, Schmid S, Krapf M, Flatz L, Born D, Jochum W, Templeton AJ, Früh M. Neutrophil-to-Lymphocyte ratio (NLR) and Platelet-to-Lymphocyte ratio (PLR) as prognostic markers in patients with non-small cell lung cancer (NSCLC) treated with nivolumab. Lung Cancer 2017;111:176-81.

5. Liu J, Li S, Zhang S, Liu Y, Ma L, Zhu J, Xin Y, Wang Y, Yang C, Cheng Y. Systemic immune-inflammation index, neutrophil-to-lymphocyte ratio, platelet-tolymphocyte ratio can predict clinical outcomes in patients with metastatic non-small-cell lung cancer treated with nivolumab. J Clin Lab Anal 2019:e22964. 
6. Minkov P, Gulubova M, Chilingirov P, Ananiev J. The Position of Neutrophils-To-Lymphocytes and Lymphocytes-To-Platelets Ratio as Predictive Markers of Progression and Prognosis in Patients with Non-Small Cell Lung Cancer. Open Access Macedonian Journal of Medical Sciences 2018;6:1382-6.

7. Zhao Z, Li X, Zhao Y, Wang D, Li Y, Liu L, Sun T, Chen $\mathrm{G}$. Role of $\mathrm{C}$-reactive protein and procalcitonin in discriminating between infectious fever and tumor fever in non-neutropenic lung cancer patients. Medicine (Baltimore) 2018;97:e11930.

8. Ni XF, Wu P, Wu CP, Ji M, Wu J, Gu XF, Jiang ZX. Elevated serum $\mathrm{C}$-reactive protein, carcinoembryonic antigen and N2 disease are poor prognostic indicators in non-small cell lung cancer. Asia Pac J Clin Oncol 2015;11:e22-30.

9. Lee JW, Seo KH, Kim ES, Lee SM. The role of (18) F-fluorodeoxyglucose uptake of bone marrow on PET/ CT in predicting clinical outcomes in non-small cell lung cancer patients treated with chemoradiotherapy. Eur Radiol 2017;27:1912-21.

10. Lee JW, Lee MS, Chung IK, Son MW, Cho YS, Lee SM. Clinical implication of FDG uptake of bone marrow on $\mathrm{PET} / \mathrm{CT}$ in gastric cancer patients with surgical resection. World J Gastroenterol 2017;23:2385-95.

11. Lee JW, Choi JS, Lyu J, Lee SM. Prognostic significance of (18)F-fluorodeoxyglucose uptake of bone marrow measured on positron emission tomography in patients with small cell lung cancer. Lung Cancer 2018;118:41-7.

12. Jiménez-Bonilla JF, Quirce R, Martinez-Rodriguez I, Banzo I, Rubio-Vassallo AS, Del Castillo-Matos R, Ortega-Nava F, Martinez-Amador N, Ibanez-Bravo S, Carril JM. Diagnosis of recurrence and assessment of postrecurrence survival in patients with extracranial non-small cell lung cancer evaluated by 18F-FDG PET/CT. Lung Cancer 2013;81:71-6.

13. Tauhardt E, Reissig A, Winkens T, Freesmeyer M. Early detection of disease progression after palliative chemotherapy in NSCLC patients by (18)F-FDG-PET. Nuklearmedizin 2014;53:197-204.

14. Jeong YH, Lee CK, Jo K, Hwang SH, Cha J, Lee JW, Yun M, Cho A. Correlation Analysis and Prognostic Impact of 18F-FDG PET and Excision Repair CrossComplementation Group 1 (ERCC-1) Expression in Non-Small Cell Lung Cancer. Nucl Med Mol Imaging 2015;49:108-14.

15. Zhou M, Chen Y, Liu J, Huang G. A predicting model of bone marrow malignant infiltration in 18F-FDG PET/CT images with increased diffuse bone marrow FDG uptake. J Cancer 2018;9:1737-44.

16. Lee JW, Jeon S, Mun ST, Lee SM. Prognostic Value of Fluorine-18 Fluorodeoxyglucose Uptake of Bone Marrow on Positron Emission Tomography/Computed Tomography for Prediction of Disease Progression in Cervical Cancer. Int J Gynecol Cancer 2017;27:776-83.

17. Inoue K, Goto R, Okada K, Kinomura S, Fukuda H. A bone marrow F-18 FDG uptake exceeding the liver uptake may indicate bone marrow hyperactivity. Ann Nucl Med 2009;23:643-9.

18. Lee JW, Na JO, Kang DY, Lee SY, Lee SM. Prognostic Significance of FDG Uptake of Bone Marrow on PET/ CT in Patients With Non-Small-Cell Lung Cancer After Curative Surgical Resection. Clin Lung Cancer 2017;18:198-206.

19. Roxburgh CS, McMillan DC. Role of systemic inflammatory response in predicting survival in patients with primary operable cancer. Future Oncol 2010;6:149-63.

20. Tolia M, Tsoukalas N, Kyrgias G, Mosa E, Maras A, Kokakis I, Liakouli Z, Kouvaris JR, Liaskonis K, Charalampakis N, Pistevou-Gombaki K, Kelekis N, Kouloulias V. Prognostic Significance of Serum Inflammatory Response Markers in Newly Diagnosed Non-Small Cell Lung Cancer before Chemoirradiation. Biomed Res Int 2015;2015:485732.

21. Balkwill F, Mantovani A. Inflammation and cancer: back to Virchow? Lancet 2001;357:539-45.

22. Scilla KA, Bentzen SM, Lam VK, Mohindra P, Nichols EM, Vyfhuis MA, Bhooshan N, Feigenberg SJ, Edelman MJ, Feliciano JL. Neutrophil-Lymphocyte Ratio Is a Prognostic Marker in Patients with Locally Advanced (Stage IIIA and IIIB) Non-Small Cell Lung Cancer Treated with Combined Modality Therapy. Oncologist 2017;22:737-42.

23. Goubran HA, Stakiw J, Radosevic M, Burnouf T. Platelets effects on tumor growth. Semin Oncol 2014;41:359-69.

24. Sullivan KM, Groeschl RT, Turaga KK, Tsai S, Christians KK, White SB, Rilling WS, Pilgrim CH, Gamblin TC. Neutrophil-to-lymphocyte ratio as a predictor of outcomes for patients with hepatocellular carcinoma: a Western perspective. J Surg Oncol 2014;109:95-7.

25. Lee JW, Ban MJ, Park JH, Lee SM. Effect of F-18 Fluorodeoxyglucose Uptake by Bone Marrow on the Prognosis of Head and Neck Squamous Cell Carcinoma. J Clin Med 2019;8:1169.

26. Lee JW, Baek MJ, Ahn TS, Lee SM. Fluorine-18- 
fluorodeoxyglucose uptake of bone marrow on PET/CT can predict prognosis in patients with colorectal cancer after curative surgical resection. Eur J Gastroenterol Hepatol 2018;30:187-94.

27. Takahashi Y, Horio H, Hato T, Harada M, Matsutani N, Morita S, Kawamura M. Prognostic Significance of Preoperative Neutrophil-Lymphocyte Ratios in Patients with Stage I Non-small Cell Lung Cancer After Complete Resection. Ann Surg Oncol 2015;22 Suppl 3:S1324-31.

28. Lim W, Ridge CA, Nicholson AG, Mirsadraee S. The 8(th) lung cancer TNM classification and clinical staging system: review of the changes and clinical implications. Quant Imaging Med Surg 2018;8:709-18.

Cite this article as: $\mathrm{Li}$ TC, Wang LL, Liu BL, Hong JJ, Xu $\mathrm{NN}$, Tang K, Zheng XW. Association between bone marrow fluorodeoxyglucose uptake and recurrence after curative surgical resection in patients with T1-2N0M0 lung adenocarcinoma: a retrospective cohort study. Quant Imaging Med Surg 2020;10(12):2285-2296. doi: 10.21037/qims-19-962
29. Mattonen SA, Davidzon GA, Benson J, Leung ANC, Vasanawala M, Horng G, Shrager JB, Napel S, Nair VS. Bone Marrow and Tumor Radiomics at (18)F-FDG PET/ CT: Impact on Outcome Prediction in Non-Small Cell Lung Cancer. Radiology 2019;293:451-9.

30. Fujima N, Hirata K, Shiga T, Yasuda K, Onimaru R, Tsuchiya K, Kano S, Mizumachi T, Homma A, Kudo K, Shirato H. Semi-quantitative analysis of pre-treatment morphological and intratumoral characteristics using (18) F-fluorodeoxyglucose positron-emission tomography as predictors of treatment outcome in nasal and paranasal squamous cell carcinoma. Quant Imaging Med Surg 2018;8:788-95. 